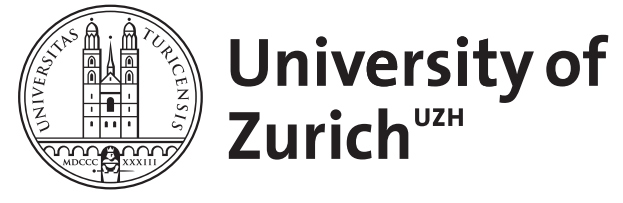

\title{
Ein Blick in die Blase-der Mensch dahinter
}

Hauri, D

\begin{abstract}
Der Blick ins Innere des Menschen ist ein alter Wunsch in der Medizin. Die Entwicklung der Zystoskopie ist den dafür Interessierten bekannt. Nachfolgend steht diese Untersuchung nicht im Zentrum des Blickpunktes, sondern die Menschen, die dahinter stehen, die ihre Energien und ihre Phantasie einsetzen, um das einmal Erreichte zu verbessern. Dabei kommt man nicht darum herum, über Menschliches-Allzumenschliches - zu berichten
\end{abstract}

DOI: https://doi.org/10.1007/s00120-004-0760-x

Posted at the Zurich Open Repository and Archive, University of Zurich ZORA URL: https://doi.org/10.5167/uzh-155807

Journal Article

Published Version

Originally published at:

Hauri, D (2005). Ein Blick in die Blase - der Mensch dahinter. Der Urologe. Ausg. A, 44(4):401-407. DOI: https://doi.org/10.1007/s00120-004-0760-x 
Urologe [A] 2005 $\cdot 44: 401-407$ DOI 10.1007/s00120-004-0760-x

(c) Springer Medizin Verlag 2005

\section{Redaktion}

P. Rathert, Düren

F.H. Moll, Köln

D. Schultheiss, Hannover
Online publiziert: 2. Februar 2005

D. Hauri · Urologische Klinik, Universitätsspital, Zürich, Schweiz

\section{Ein Blick in die Blase - der Mensch dahinter}

Mainz auf die Welt. Sein Vater war anlässlich eines fatalen Duells gezwungen nach Deutschland zu fliehen. Philipp begann sein Medizinstudium in Mainz, musste jedoch nach der vorläufigen Auflösung der dortigen Universität anlässlich des Einmarsches der Franzosen 1794 nach Jena umsiedeln. Er ist dort u. a. auch Schüler von Christoph Wilhelm Hufeland, einem der fruchtbarsten Wissenschafter und Schriftsteller seiner Zeit, in dessen medizinischem Journal später die Erstveröffentlichung seines „Lichtleiters“ herauskommen sollte [3]. Bozzini promovierte 1796 zum Doktor der Medizin und ließ sich als praktischer Arzt in der Stadt Mainz nieder. Da Mainz im Frieden von Luneville 1801 nach dem II. Koalitionskrieg zwischen $\mathrm{Na}$ poleon und Kaiser Franz an Frankreich abgetreten wurde und Bozzini nicht als Franzose eingebürgert werden wollte, siedelte er nach Frankfurt über. Dort erhielt er wegen verschiedenster übler Intrigen lange Zeit das Bürgerrecht nicht, was ihn an der praktischen Ausübung der ärztlichen Tätigkeit hinderte [4].

Nachdem schon 1805 eine kurze Annonce im „Reichsanzeiger“ gedruckt wurde (• Abb. 2), erschien die ausführliche Erstbeschreibung seines „Lichtleiters“ 1 Jahr später [3]. Es war der typische Ausdruck des Wechsels von der damals noch allgemein verbreiteten vitalistischen naturphilosophischen Medizin zugunsten einer physiologischen Denkweise.

Die Konstruktionsweise des Lichtleiters ist allgemein bekannt (• Abb. 3): Die vasenförmige, von einer Kerze beleuchtete Laterne aus lederbezogenem Blech, welche durch eine vertikale Wand unterteilt war. Durch die eine Hälfte des Lichtge- fäßes konnte das Innere des Körpers ausgeleuchtet werden, währenddem der Beobachter durch ein Loch in der 2. Hälfte mittels Linsen und verschieden geformter Silberröhren das Innere erforschen konnte.

Bozzini wollte nicht nur die Blase inspizieren, sondern v. a. die besser zugänglichen Organe wie Larynx, Vagina und Darm. Er stellte seine Erfindung Erzherzog Karl, dem Bruder des österreichischen Kaisers, Franz dem Ersten, vor, den er als Teilnehmer des II. Koalitionskrieges (1798-1802) kennen gelernt hatte. Bozzini diente in der kaiserlichen Feldar-

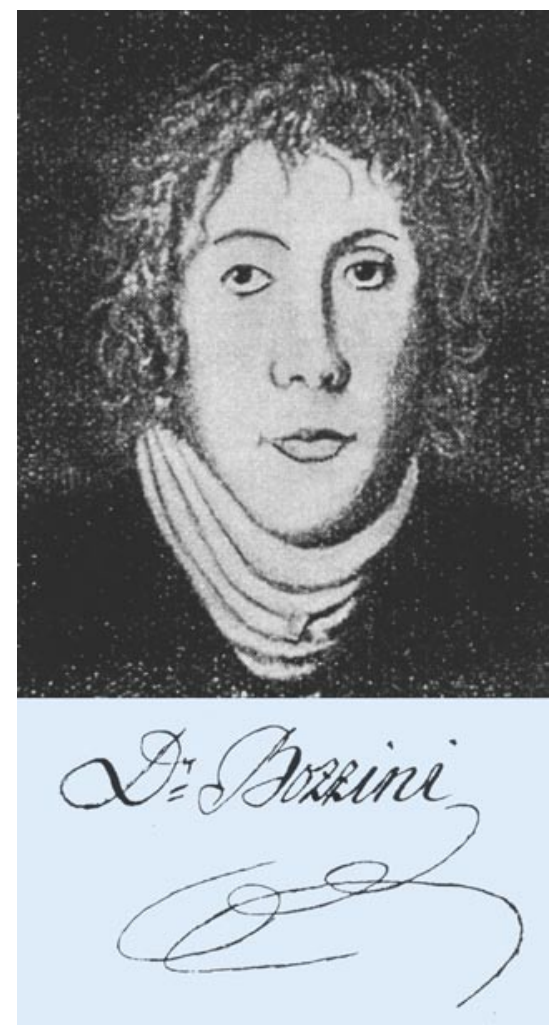

Abb. 1 A Philipp Bozzini (1773-1809)
Aus einer vornehmen italienischen Fami lie stammend kam Bozzine (• Abb. 1) in 
$\mathbf{A}$

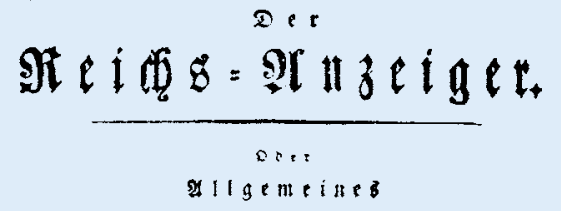

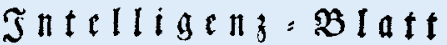 in un $B$ cesuf

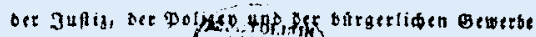
im $\mathfrak{s}$ (4t) ntrard

fut bfentiden untefbartang bes qefet ibte

gameinnúgige Begengande alter $x$ is.

\begin{tabular}{|c|}
\hline 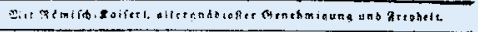 \\
\hline $\begin{array}{c}\text { 3atra a } 1805 . \\
\text { erpet }\end{array}$ \\
\hline
\end{tabular}

B

Rinfle, Manufacturen uno Fabrifen.

Gerwiffe IIrfaden beftimmen mid, ofe Injerge von ber Erfinoung eluer tikfaden uno leithe antrentbaren. Bortidenng in mas cen, ourd bie mant in alle innere Soblen bes Iebenden antmalifaten Rerpers, ole nibt

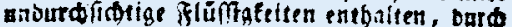
phufiologifice ooer paibchogifte Deffinung gei nala fobert falin. Sie itt Demfilben vorgegens ben functionen eríbeinert oem xage eben fo beutlid, als auf oer Doerfighe, und is lafis fen. In Sperationen in then incer thumen. bung bes Sefintofinned vornebmen. Jobs:

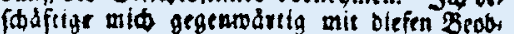
adrungen, uno werbe fie nebfl ber Bortidy tung in ser folge befanit maden.

D. Boyjuri,

Ifrjt und Beburtibelfer ju Brfft.

a. S.

Abb. $2 \triangle$ Annonce im „Reichsanzeiger" 1805 über die Erfindung des "Lichtleiters"

Abb. 5 Marie Alexandre Désormeaux, Geburtshelfer und Vater von Antonin Jean bei einer Entbindung zu Zeiten der französischen Revolution

mee als Regimentsarzt und leitete in dieser Funktion ein 120 Betten starkes Feldlazarett. Er erhielt von seinem direkten Vorgesetzten ein vorzügliches Zeugnis [5]. Dem Erzherzog war die Tragweite der Erfindung einsichtig und er leitete die Beschreibung zur Überprüfung nach Wien weiter, um das Instrument an den österreichischen Militärspitälern einzusetzen. Hierzu war ein Gutachten der obersten Sanitätsbehörde notwendig und das war die 1785 von Josef dem Zweiten gegründete Medizinisch-Chirurgische Josefs-Aka-

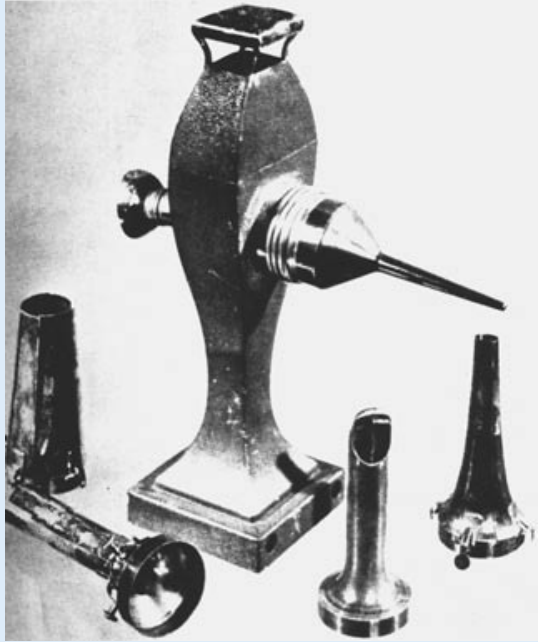

Abb. $3 \triangle$ Bozzinis „Lichtleiter"

Abb.4 Antonin Jean Désormeaux (1815-1882)
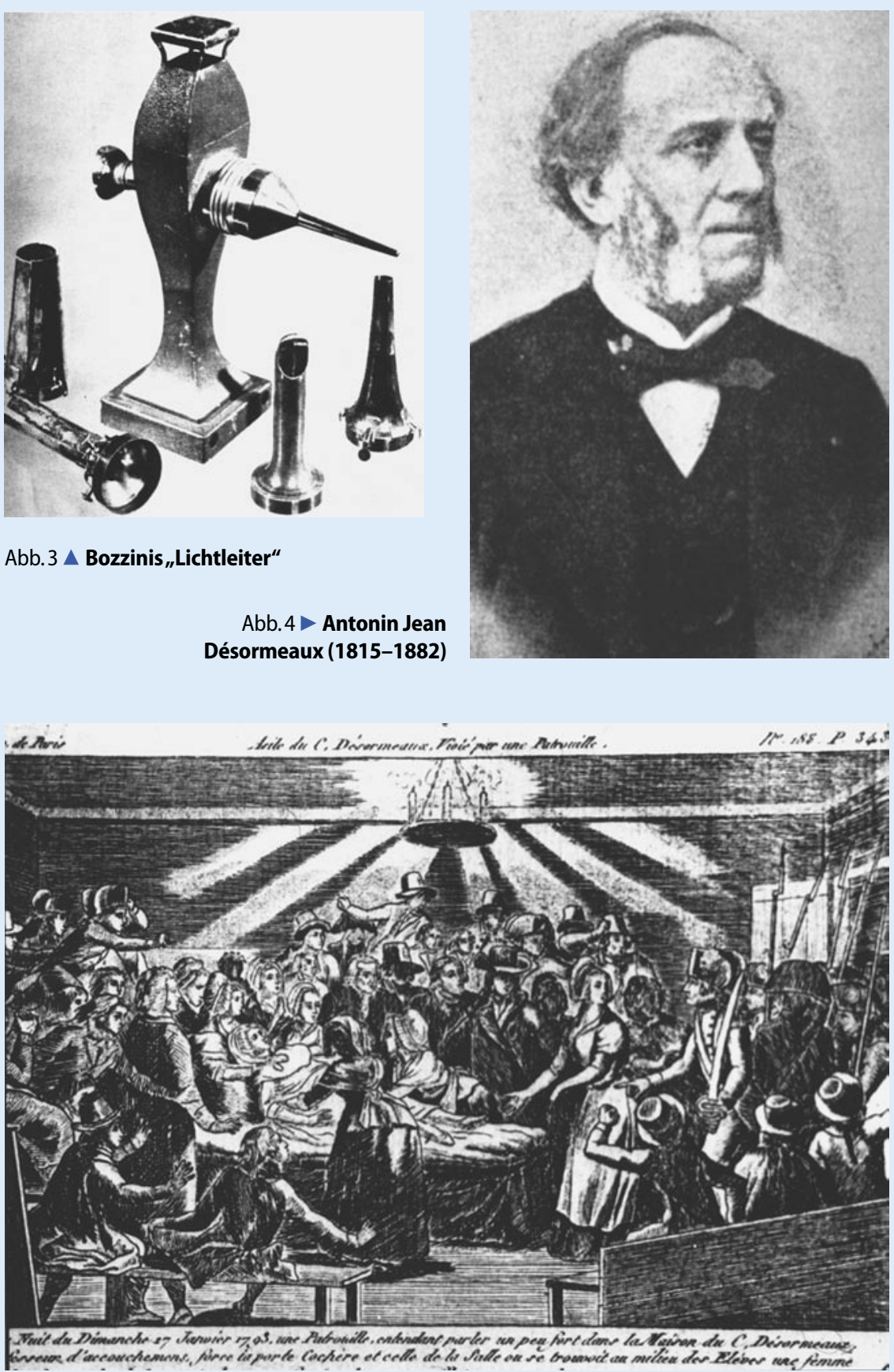

demie. Damit geriet Bozzini in den gefährlichen Strudel der österreichischen Innen- und Standespolitik, deren Sprengkraft er als Außenstehender nicht vorhersehen konnte.

Die Josefs-Akademie wurde als Symbol für die Aufwertung der Chirurgie in der Donaumonarchie seit ihrer Gründung von Wiener Ärzten heftig bekämpft. Hauptgegner waren die Mitglieder der Wiener Medizinischen Fakultät. Neben dem Ressentiment der akademischen Ärzte gegenüber den „Feldschern und Barbieren“ gab es eine, jeder Neuerung abholden Fraktion, deren einflussreichster Repräsentant Andreas Josef Freiherr von Stifft (176o-1836) war, gleichzeitig Leibarzt des Kaisers und als Protomedicus der Erblande oberster Dienstherr der Medizinischen Fakultät. Ihm musste als grundsätzlichem Gegner aller Neuerungen ein Instrument wie der Lichtleiter revolutionär vorkommen [6].

Die Josefs-Akademie zeigte sich schon von der Beschreibung des Lichtleiters so angetan, dass sie ein Exemplar kaufte und damit Untersuchungen durchführte. Aus 


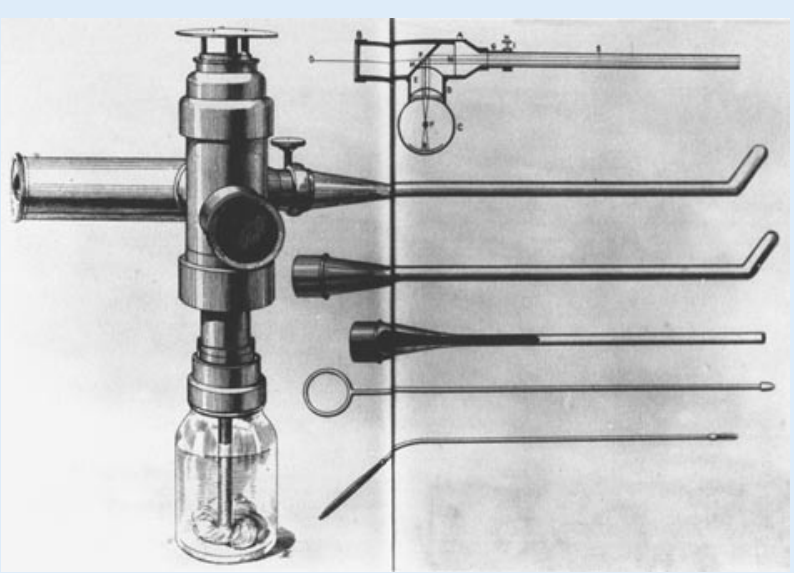

Abb. $6 \Delta$ „Endoscope“ von Désormeaux

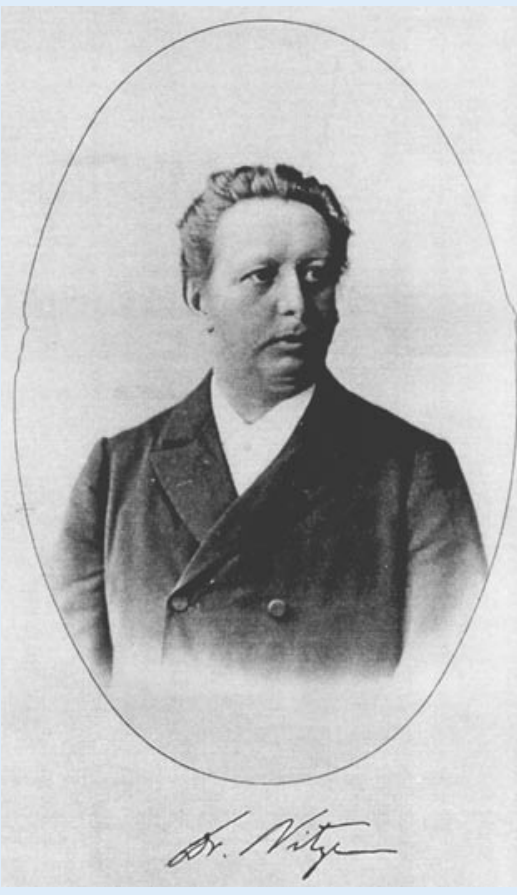

den Protokollen geht die Tauglichkeit des Lichtleiters eindeutig hervor. Zunächst untersuchte man an Leichen Blase, Mastdarm und Scheide. Mittels kleiner Schnitte wurde das Instrument in die Bauchhöhle eingeführt und sowohl das Bauchfell wie der Darm betrachtet. Danach setzte die Kommission die Untersuchungen mit Ausnahme der Schnittöffnungen an Patienten fort und beschrieb ebenfalls verwertbare Ergebnisse. Besonders erwähnenswert ist die fast völlige Schmerzlosigkeit der Methode. Die Kommission betonte die Entwicklungsfähigkeit der Erfindung und verlangte gleichzeitig verschiedene Änderungen. In der Folge beauftragte sie den Prosektor Ilg, die Vorschläge zu realisie-

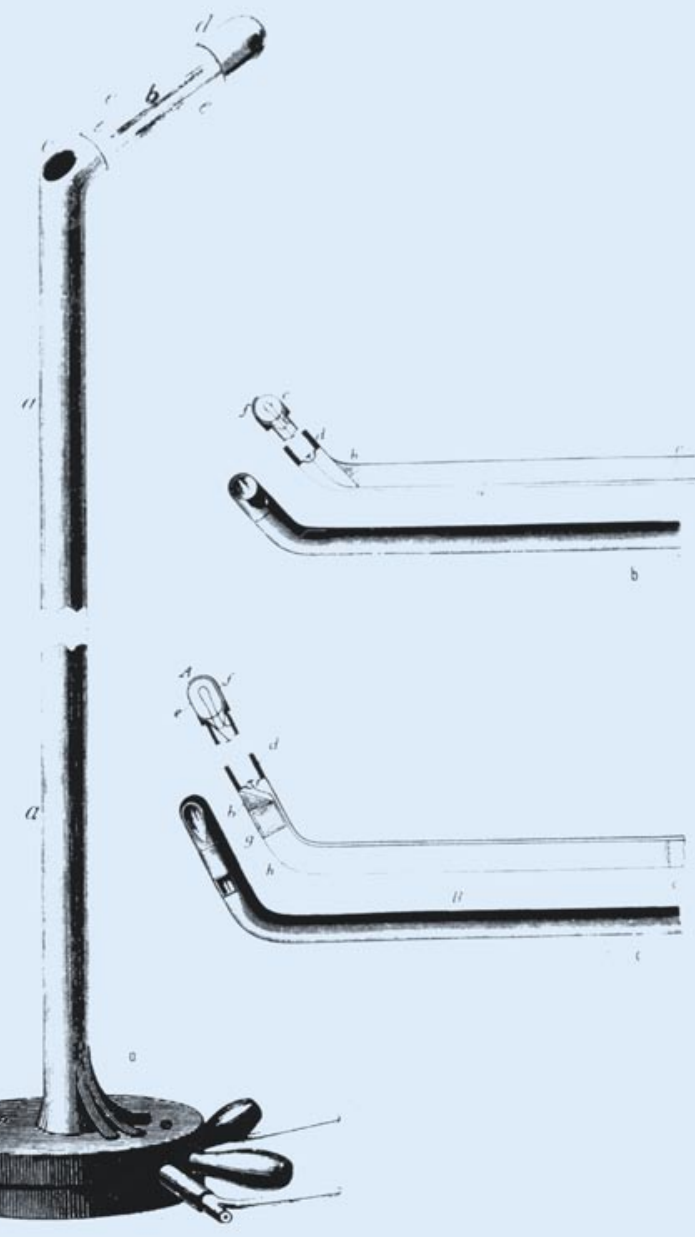

Abb. $8 \Delta$ Erstes Nitze-Leiter-Zystoskop
Abb.7 $<$ Maximilian Nitze

(1848-1906) ren und wiederholte die Untersuchungen mit dem verbesserten Instrument. Die Ergebnisse waren derart überzeugend, dass der Erzherzog dem Erfinder eine goldene Ehrenmedaille verleihen wollte. Hier griff der kaiserliche Leibarzt entscheidend ein. Er empfand das Gutachten als Affront gegen die Fakultät und Prestigeverlust seiner Person. Zwar wagte er keinen offenen Widerspruch gegen den Erzherzog; er erreichte aber innerhalb Wochenfrist die Erstellung eines 2. Gutachtens von der Medizinischen Fakultät.

Der Bericht musste dem Kaiser selbst vorgelegt werden. Dieses erstaunliche Detail lässt mit Sicherheit den Schluss zu, dass der Monarch den Einflüsterungen seines Leibarztes folgte, denn anders erklärt sich nicht, wieso er sich mitten im Krieg derart um die Prüfung eines für ihn letztlich peripheren medizinischen Geräts kümmerte, dessen Funktion und Tragweite ihm als medizinischem Laien sicher verborgen bleiben musste. Das Gutachten der Medizinischen Fakultät, hinter welchem auch die katholische Kirche stand, fiel erwartungsgemäß vernichtend aus, und wurde bezeichnenderweise nicht in einer Fachzeitschrift, sondern 1807 in der „Wiener Zeitung “ veröffentlicht. Bozzini erhielt einen Verweis erteilt mit den Worten: „es wäre eine Vermessenheit und Sünde, allenfalls mit dieser Maschine eine Zervix während eines Orgasmus zu beobachten, und 
gleichzeitig eine Verwegenheit mit einem solch gefährlichen Instrument in solch verborgene Regionen vorzudringen, welches von der Menschheit nicht akzeptiert werden könne“.

Die Polemiken in deutschen und österreichischen Publikationen wurden nach dem Bekanntwerden des Fakultätsgutachtens verschärft fortgesetzt. Verbittert starb der an sich weltoffene Bozzini 1809, 36-jährig, unerwartet an einer Typhusepidemie. Seine 1954 restaurierte Grabplatte kann heute noch an der Außenwand des Doms zu Frankfurt am Main besucht werden. Der Traum und die Bestrebung, in das Innere des Menschen zu schauen, aber lebte weiter.

\section{Antonin Jean Désormeaux (1815-1882)}

Désormeaux (• Abb. 4) weist eine lange Ahnengalerie von Ärzten auf [7]. Schon sein Urgroßvater, praktizierender Arzt in Bloix, war der Sohn eines Chirurgen. Sein Großvater unterrichtete in Paris als Geburtshelfer. Dessen Sohn, Marie-Alexandre, der Vater von unserem als Urologe und Chirurg bekannten Antonin-Jean Désormeaux, war Chirurg und Geburtshelfer, Chefarzt an der Maternité, und Nachfolger von Beaudeloc, dessen Name diese Klinik noch heute trägt. Dieser, sein Vater, lebte in den stürmischen Zeiten der französischen Revolution. In den Annalen des Spitals kann man Folgendes nachlesen [8]: „Sonntagnacht, am 27. Januar 1791, stürmte plötzlich eine Streife in das Haus von Doctor Désormeaux, Professor für Geburtshilfe, während dieser gerade im Beisein seiner Schüler und mehrerer Hebammen eine Frau entband. Die Oberhebamme gab dem Kommandanten eine Ohrfeige, worauf sich der Trupp wieder verzog“ (- Abb. 5).

Désormeaux ersetzte die Kerze Bozzinis als zu schwache Lichtquelle durch eine Flamme aus einem Gemisch von Petrol, Alkohol und Terpentin, weshalb seine Apparatur einen Kamin benötigte (• Abb. 6). Er übernahm die ursprüngliche Idee von Ségalas [9] und leitete das Licht über einen um $45^{\circ}$ geneigten Spiegel, mit einer zentralen Öffnung, wodurch über Sonden mit einem Durchmesser von 2-8 mm die körperinneren Organe ausgeleuchtet werden konnten [10].

Das „Endoskop“ - der Name wurde erstmals von Désormeaux eingeführt wurde von Charrière gebaut. 9 Jahre nach der ersten Präsentation seines Endoskops vor der "Académie de Médecine“ in $\mathrm{Pa}$ ris wurde ihm 1855 der prestigeträchtige „Prix du Marquis d'Argenteuil“ in der Höhe von 12.0oo Francs verliehen [11]. Désormeaux beschrieb mit Hilfe seiner Apparatur Blasen- und Harnröhrenschleimhautveränderungen. Er konnte Blasensteine erkennen und diese ausmessen. Er führte innere Urethrotomien durch und es gelang ihm auch, ein „Urethrapapillom“ zu erkennen und dieses zu koagulieren [12]. Er war auch einer der Ersten, der mit seinem Instrument Rektoskopien durchführte. Schon früh befasste er sich mit der Idee, die Lichtquelle in das Blaseninnere zu verlegen. Interessanterweise zeigte der zu gleicher Zeit wirkende Civiale (1792-1867) kein Interesse an der neuen Möglichkeit der Endoskopie.

Das Instrument war jedoch schwierig zu handhaben und nur wenige ahnten seine Zukunft. Einer seiner Lehrer äußerte sich einmal Désormeaux gegenüber: „Man sieht sehr gut mit ihrem Instrument. Aber wozu dient das eigentlich?“ [10]. Und so gerieten das „Endoskop“ und mit ihm sein Erfinder für einige Zeit in Vergessenheit.

\section{Maximilian Nitze (1848-1906)}

Nitze (• Abb. 7) gelang es als Erstem, den lange gehegten Wunsch nach der Lichtquelle innerhalb der Blase zu verwirklichen. Nach dem Medizinstudium in Heidelberg, Würzburg und Leipzig bildete sich Nitze an verschiedenen Krankenhäusern in Dresden weiter. Dort erfuhr er offensichtlich von der Anwendung des glühenden Platindrahtes zur Beleuchtung innerer Körperhöhlen, an welchem der Breslauer Zahnarzt Julius Bruck (1840-1902) experimentierte. Bruck entwickelte diese Lichtquelle für die Stomatologie. Aus welchen Gründen auch immer hätte er auch gerne die Blase ausgeleuchtet. Das hellste Licht, das man damals erzeugen konnte, war der glühende Platindraht, der jedoch wegen seiner Hitze zu Schleimhautverbrennungen führte. Er schloss deshalb diesen Platindraht in eine doppelte Glas$\frac{\text { Hier steht eine Anzeige }}{\text { This is an advertisement }}$

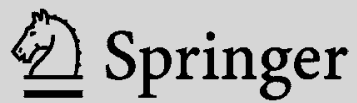


flasche ein, deren äußere Kammer dauernd mit Eiswasser gespült wurde. Das Gerät wurde dadurch so voluminös, dass er die Blase lediglich durchleuchten konnte, indem er dieses Gerät in den Enddarm einbrachte. Mittels eines gebogenen Katheters, an dessen konvexem Teil der Krümmung eine plane Glasscheibe eingelassen war, konnte er so die vom Enddarm her beleuchtete Blase beobachten [13].

Mit seinem ersten Instrumentenbauer Wilhelm Deicke konstruierte Nitze sein Charrière-21-Zystoskop. Revolutionär daran war einmal die erstmals mögliche intravesikale Ausleuchtung mittels des glühenden Platindrahtes, wozu eine Wasserspülung notwendig war und anderseits der zwischengeschalteten Linsen wegen zwecks Vergrößerung des Gesichtsfeldes. Allerdings war das erhaltene Bild 2-mal seitenverkehrt, was Nitze offensichtlich nicht störte. Am 2. Oktober 1877 demonstrierte er sein Zystoskop vor der Königlichen Medizinischen Gesellschaft in Dresden an einer Leiche, wobei es ihm gelang, einen in die Harnblase eingebrachten Stein richtig zu erkennen. Für die Weiterentwicklung zur klinischen Anwendung war Deicke jedoch überfordert. Nitze zog daher nach Wien zu Josef Leiter (1830-1892), einem der damals bekanntesten und fähigsten Instrumentenbauer. Am 9. März 1879 konnte er vor der Königlichen Medizinischen Gesellschaft in Wien das erste Nitze-Leiter-Zystoskop am Menschen demonstrieren (• Abb. 8). Am 14. Juni 1879 erschien der erste Bericht in der Wiener Medizinischen Wochenschrift [14].

Das Gerät wurde vom anwesenden Ärztegremium begeistert empfangen. Doch bald erhob sich auch öffentliche Kritik: einerseits wegen des recht hohen Preises des Instrumentes und dann wurde weiterhin über den Sinn solcher Untersuchungen heftig diskutiert [15]. Doch bald zerstritt sich Nitze mit seinem Instrumentenbauer. Josef Leiter (• Abb. 9), Sohn eines unbemittelten Militärschuhmachermeisters, konnte nur 3 Jahre Schulausbildung genießen und wurde in der Folge als chirurgischer Instrumentenmacher ausgebildet [16]. Seine Wanderschaft führte ihn auch nach Paris, wo die damals berühmtesten Instrumentenmacher wirkten. Unter anderem ging er auch bei Charrière, einem gebürtigen Schweizer [17], in die Lehre. Die französischen Instrumentenmacher, so auch Leiter, verstanden sich als den Chirurgen gleichgestellte Partner und nicht als Befehlsempfänger wie in Deutschland. Dies ertrug Nitze offensichtlich nicht. Zudem gab es patentrechtliche Streitigkeiten $[25,26,27,28]$. Nitze verließ 188 o Wien und ließ sich in Berlin als praktizierender Blasenfacharzt nieder. Seine Praxis wurde zu einem Mekka für Endoskopie. Wegen seiner unbestreitbaren Verdienste wurde er 1889 zum außerordentlichen Professor für Urologie an der Universität Berlin ernannt.

1879/1880 erfand Thomas A. Edison die Glühbirne. Valentine in New York gelang es 8 Jahre später diese zu miniaturisieren, sodass sie als sog. „Mignon-Lämpchen" in die Endoskope eingebaut werden konnte, was eine deutliche Verbesserung bedeutete $[18,19]$. So war es Nitze mit den Jahren möglich, mittels eines vom Instrumentenmacher P. Hartwig in Berlin eigens für Nitze konstruierten Operationszystoskops kleinere Blasenoperationen durchzuführen [20, 21]. Später gelang es ihm Blasenbefunde fotografisch festzuhalten [22]. Und weitere Jahre später führte er die ersten Harnleiterkatheterisierungen $\mathrm{zu}$ diagnostischen Zwecken durch [23, 24].

So genial das Werk Nitzes heute beurteilt wird, muss man sich jedoch bewusst sein, dass diese Entwicklung im Zuge der Zeit lag. Nitze hatte einen schwierigen Charakter. Er war introvertiert, eifersüchtig, herrschsüchtig und neigte rasch zu Streitereien. Er weigerte sich kaufmännische und patentrechtliche Probleme anzugehen, sodass er sich immer wieder mit seinen Instrumentenmachern überwarf. Er war auch nicht bereit, sein Wissen und Können seinen Schülern weiterzuvermitteln. Es fehlte ihm das Interesse, mit guten Optikern, die es damals zweifellos gab, zusammenzuarbeiten. Unwillig löste er sich auch vom Platindraht und akzeptierte erst auf Druck von v. Dittel in Wien, das Mignon Lämpchen in seine Instrumente einzubauen. Mit seinem Charakter hatte er laut Berichten von Zeitzeugen lange die Gründung der Deutschen Gesellschaft für Urologie zu verhindern vermocht $[25,26$, 27, 28], kurz: Nitze war ein eigenbrötlerischer Tüftler - ein Genie war er nicht.
Urologe [A] 2005 - 44:401-407

DOI 10.1007/s00120-004-0760-x

(c) Springer Medizin Verlag 2005

\section{Hauri}

Ein Blick in die Blase der Mensch dahinter

\section{Zusammenfassung}

Der Blick ins Innere des Menschen ist ein alter Wunsch in der Medizin. Die Entwicklung der Zystoskopie ist den dafür Interessierten bekannt. Nachfolgend steht diese Untersuchung nicht im Zentrum des Blickpunktes, sondern die Menschen, die dahinter stehen, die ihre Energien und ihre Phantasie einsetzen, um das einmal Erreichte zu verbessern. Dabei kommt man nicht darum herum, über Menschliches - Allzumenschliches $-\mathrm{zu}$ berichten.

\section{Schlüsselwörter}

Zystoskopie · Philipp Bozzini .

Antonin Jean Désormeaux .

Maximilian Nitze $\cdot$ Harald Hopkins

\section{A look inside the bladder - the human behind it}

\section{Abstract}

To study the insides of humans is an old wish of mankind. Cystoscopy and its development are known toinitiates. In the following we do not stress this examination, but focus on those people behind it who shared in paving the way for improvement with their fantasy and energy without neglecting to report on the human and very human aspects.

Keywords

Cystoscopy · Philipp Bozzini ·

Antonin Jean Désormeaux .

Maximilian Nitze · Harald Hopkins 


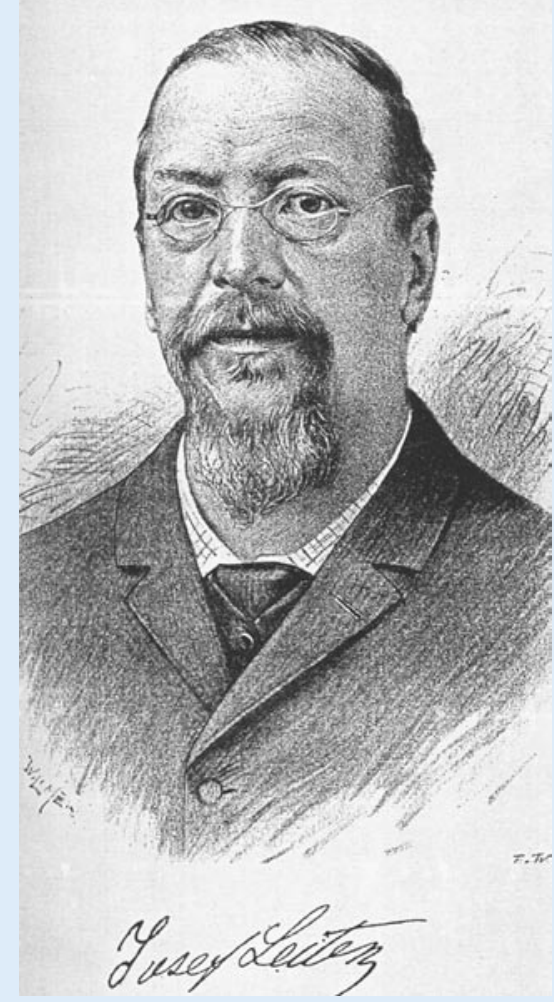

Abb. $9 \triangle$ Josef Leiter (1830-1892)
0

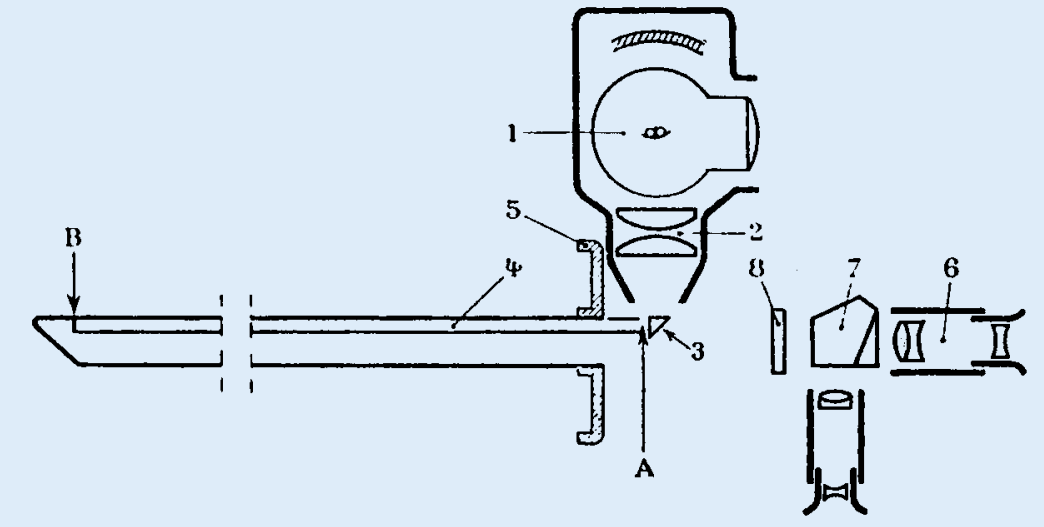

Abb. $10 \Delta$ Die äußere Lichtquelle mit Lichttransmission über einen Prismastab nach Fourestier

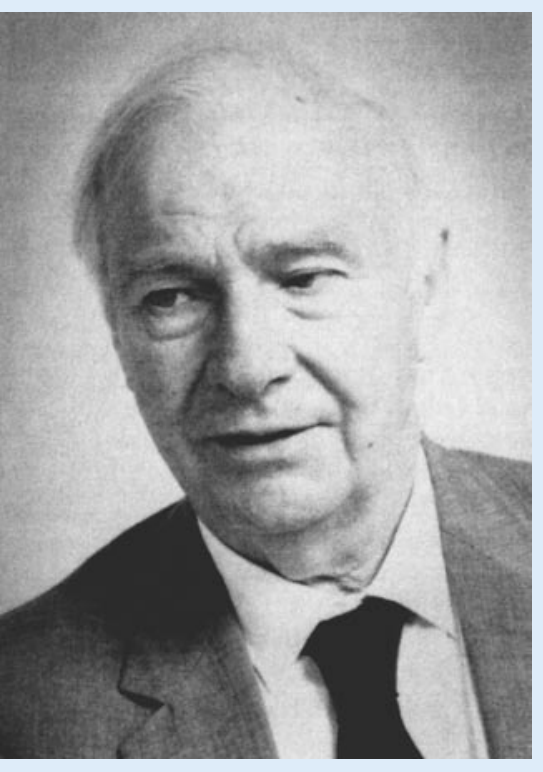

Abb. $13>$ Erste durch ein

Fiberskop entstandene Fotographie einer amerikanischen Briefmarke, 1957 [34]

(- Abb. 10). Er war damit im Stande, die ersten endoskopischen Filme zu projizieren. Das Prinzip konnte sich aber nicht durchsetzen, weil es sehr teuer war, der Prismastab leicht brach und das ganze Instrumentarium für operative Eingriffe viel zu unhandlich war.

Da erinnerte man sich an ein Vorbild aus der Natur: Das Auge gewisser Insekten wie z. B. Fliegen und Bienen. Deren Augen sind aus einer großen Anzahl lichtempfindlicher Stabzellen zusammengesetzt, deren Weiterleitung in das Zentralnervensystem schlussendlich ein aus Punkten zusammengesetztes Ganzes ergibt. Bei der Endoskopie müsste diese Art von Lichtübertragung zusätzlich in

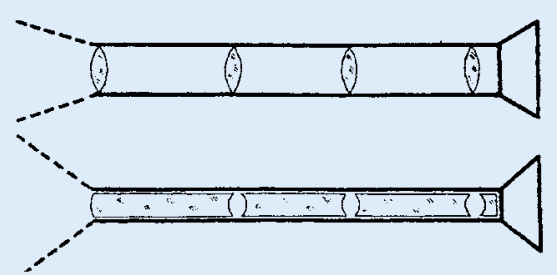

Abb. $12 \Delta$ Oben traditionelle Optik. Unten Hopkins-Optik (s. Text)

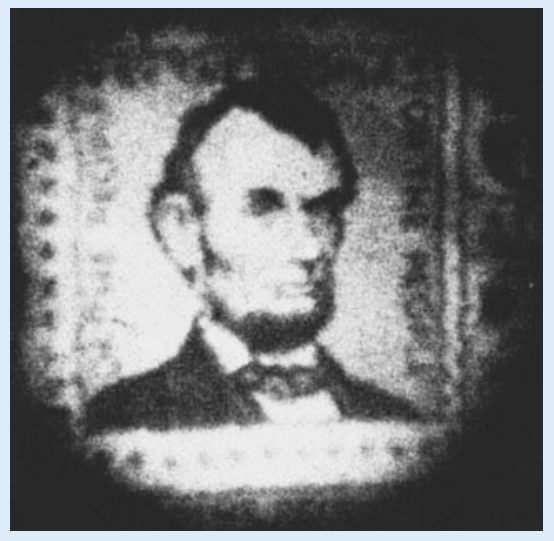

flexibler Form möglich sein. Dem dänischen Ingenieur Holger Møller Hansen gelang es anfangs der 5oer Jahre nachzuweisen, dass ein Faden von 1 mm Durchmesser aus dem Plastmaterial Perspex (Methylmetacrylat) Lichtwellen wie Stäbchen aus Glas leiten können. Da die Lichtwellen die Wand des Materials durchdrangen, litt jedoch die Bildqualität. Mangels finanzieller Quellen mussten die Experimente abgebrochen werden [30].

Und dann war es Zeit für Harold Hopkins, (• Abb. 11) einen der genialsten Physiker seiner Zeit. Sein Interesse wurde anlässlich eines 1951 durchgeführten Gastroenterologen-Kongresses geweckt, wo er sich über die miserable Bildqualität wun- 
derte und von den Kollegen gedrängt wurde, Ideen zur Verbesserung der Lichtqualität zu entwerfen [31]. Schon 3 Jahre später publizierte er in Nature seine revolutionäre Idee und damit war die Fiberglaslichtübertragung geboren [32]. Sie beruht darauf, dass die einzelnen Glasfasern mit einer 2. Schicht eines tieferen Refraktionsindex bedeckt sind, wodurch der Lichtstrahl im Kern einen Zickzackkurs durch Reflexion am Mantel einnimmt und so an Energie gewinnt. Das Prinzip wurde 1956 patentiert. Hopkins erhielt jedoch keine finanzielle Unterstützung, sodass weitere Forschung und Vermarktung nach Ann Arbour/USA wechselten.

Hopkins zog sich resigniert zurück und wieder war es ein Zufall, der zu seiner zweiten genialen Erfindung führte [33]: einige Urologen in Liverpool hatten den Eindruck, dass die Blasenkarzinomfälle sich häuften. Sie hätten ihre Entwicklung gerne fotographisch dokumentiert. Das war mit den damaligen Linsensystemen unmöglich. Hopkins war in der Lage, mit einem Stipendium von damals 3000 Pfund und innerhalb von 18 Monaten die noch heute verwendeten Optiken zu präsentieren (• Abb. 12). Währenddem die bisherigen Optiken im Prinzip ein Rohr aus Luft mit Glaslinsen darstellten, erfand Hopkins das System des Rohres aus Glas und der darin eingebauten Linsen aus Luft, womit er ein größeres Gesichtsfeld, eine 8o-mal höhere Lichttransmission und einen 90\%igen $\mathrm{Ge}$ winn an Lichtintensität erreichte.

Die ersten durch ein Fiberskop erhaltenen Bilder ( $\bullet$ Abb. 13) begeisterten verständlicherweise die daran Beteiligten, bedeuten für uns heute zumindest einen geglückten Schritt in der weiteren Entwicklung. Die ersten vorläufigen intravesikalen Fotographien wurden 1961 am Kongress der Internationalen Gesellschaft für Urologie in Rio de Janeiro gezeigt. Trotzdem hatte Hopkins wiederum Schwierigkeiten, indem kein angloamerikanischer Instrumentenmacher diese Optik in ein Zystoskop integrieren wollte, um es auf den Markt zu bringen. Es war ein glücklicher Zufall, dass Hopkins, der fließend deutsch sprach, anlässlich eines Kongresses in Deutschland über das Fotographieren in der Medizin auf Karl Storz traf. So konnte das erste Zystoskop für den Handel von Storz mit einer Hopkins-Optik
1967 am Kongress der Internationalen Gesellschaft für Urologie in München vorgestellt werden.

Diese revolutionären Erfindungen wären nobelpreiswürdig gewesen. Hopkins, der nebenbei das erste Zoomlinsensystem für die BBC konstruierte, war dazu viel zu bescheiden - und vielleicht auch zu intelligent. Und wie der englische Dichter Henry W. Longfellow (1807-1882) schrieb: „The heights of great men reached and kept, were not attained by sudden flight, but they, while their companions slept, were toiling upwards in the night“.

\section{Korrespondierender Autor Prof. Dr. D. Hauri}

Urologische Klinik, Universitätsspital, Frauenklinikstraße 10, CH-8091 Zürich, Schweiz E-Mail: hauri@uro.usz.ch

\section{Interessenkonflikt: Keine Angaben}

\section{Literatur}

1. Nöske H, Breitwiesen D (1973) Zur Geschichte der urologisch-endoskopischen Diagnostik. Münch Med Wochenschr 115: 1927

2. Rognon LM, Raymond G (1992) Historique de I'hyperplasie bénigne de la prostate. Ann Urol 26: 176

3. Bozzini $P$ (1806) Lichtleiter, eine Erfindung zur Anschauung innerer Theile und Krankheiten. J Pract Heilkd 24: 107-124

4. Roediger E (1972) Der Frankfurter Arzt Philipp Bozzini, der Erfinder des Lichtleiters. Med Histor J 7: 204-217

5. Reuter MA (1998) Geschichte der Endoskopie, Bd 1. Krämer, Stuttgart Zürich, S 17

6. Lesky E (1970) Die Wiener Experimente mit dem Lichtleiter Bozzinis 1806/1807. Clio Med Pergamon Press NY 5: 327-350

7. Chevassu MM (1955) Antonin Désormeaux, Père de l'endoscopie. Hist Med 5: 93-96

8. Hartemann J (1981) Geschichte der Geburtshilfe vom 18. Jahrhundert bis zur Gegenwart. Illustrierte Geschichte der Medizin, Bd 4. Andreas \& Andreas, Salzburg, S 1361

9. Ségalas M (1827) Description of an instrument for inspecting the urethra and bladder. Lancet 7: 603

10. Hillemand P, Gilbrin E (1976) Antonin Jean Désormeaux (1815-1894), le créateur de l'endoscopie. Bull Acad Nat Med 160: 95-100

11. Désormeaux MAS (1855) De l'endoscope, instrument propre à éclairer certaines cavités intérieures de l'économie. Comptes Rendus Hébdomadaires des Séances de l'Académie des Sciences 40: 692

12. Désormeaux A (1865) De l'endoscope et de ses applications au diagnostic et au traitement des affections de l'urèthre et de la vaissie. Baillière, Paris

13. Zamann AP, Zajaczkowski T (2002) Julius Bruck (1840-1902). Sein Beitrag zur Weiterentwicklung der Endoskopie. Urologe B 42: 35-39

14. Nitze M (1879) Beobachtungs- und Untersuchungsmethode für Harnröhre, Harnblase und Rectum. Wien Med Wochenschr 24: 650
15. Blum V (1929) 50 Jahre Zystoskopie. Wien Klin Wochenschr 42: 797-800

16. Reuter HJ (1997) Biographie von Josef Leiter, Instrumentenmacher Nitzes. Das Wiener Endoskopie-Museum. Literas Universitätsverlag, Wien, S 48

17. Boschung U (1985) Joseph-Frédéric-Benoît Charrière (1803-1876) Schweiz Rundschau Med Prax 74: 181-184

18. Valentine FC (1899) An improved urethroscope. Med News 74: 588

19. Nitze M (1887) Ueber Elektroendoskopie der männlichen Harnblase. Centralblatt Chir 84

20. Nitze M (1891) Das Operationscystoskop - vorläufige Mitteilung. Centralblatt Chir 51: 993

21. Nitze M (1895) Ueber intravesikale Operationen von Blasengeschwülsten. Centralblatt Chir 22: 971

22. Nitze M (1893) Zur Photographie der Harnblase. Berliner Med Wochenschr 178: 316

23. Nitze M (1896) Demonstration zum Katheterisieren der Harnleiter beim Manne. Verhandlungen Berliner Med Ges 26: 21

24. Nitze M (1895) Ueber cystoskopische Diagnostik chirurgischer Nierenerkrankungen mit besonderer Berücksichtigung des Harnleiterkatheterismus. Berl Klin Wochenschr 32: 350

25. Ringleb O (1925) Maximilian Nitzes Erfindung des Cystoskops und sein Ausbau der Gebrauchsanweisung. Z Urol 19: 484-495

26. Ringleb O (1929) Die ersten 50 Jahre der Arbeit mit dem Cystoskop. Chirurg 1: 126-29

27. Ringleb O (1932) Zur Erinnerung an Maximilian Nitze. Z Urol Chir 36: 1-15

28. Ringleb O (1939) Maximilian Nitze und die Erfindung des Cystoskops. Z Urol 33: 579-590

29. Fourestier M, Gladu A, Vulmière J (1952) Perfectionnements à l'endoscopie médicale. Press Med 60: 1292-1294

30. Lauridsen C (1997) Die Priorität zur Einführung des Fiberlichtes. Das Wiener Endoskopie-Museum. Literas Universitätsverlag, Wien, S $88 \mathrm{ff}$

31. Gow JG (1998) Harald Hopkins and optical systems for urology - an appreciation. Urology 52: 152-157

32. Hopkins HH, Kapany NS (1954) A flexible fiberscope, using static scanning. Nature 173: 39-41

33. Gow JG (1997) Hopkins' contributions to medecine. Das Wiener Endoskopie-Museum. Literas Universitätsverlag, Wien, S 67

34. Hirschowitz BI (1979) A personal history of the fiberscope. Gastroenterology 76: 864-869 